\title{
REGRA INERCIAL DO BANCO CENTRAL BRASILEIRO E EXPECTATIVAS ADAPTATIVAS DE INFLAÇÃO: O PERIODO JAN/2005-JUN/2010
}

\author{
Ricardo Ramalhete Moreira
}

\begin{abstract}
Resumo
A partir do referencial teórico das regras de Taylor, o trabalho estuda a aderência empírica de regras alternativas de política monetária na economia brasileira, no período de janeiro de 2005 a junho de 2010. Os principais resultados apontam para: a) presença de forte inércia do nível da taxa Selic; b) expectativas de inflação como variável altamente explicativa na formação dos níveis e das variações da taxa Selic, sugerindo comportamento forward-looking do Banco Central; c) gap do produto com baixo poder de explicação nas decisões de política monetária; d) expectativas de inflação com forte componente adaptativo no período amostral.
\end{abstract}

Palavras-chave: Expectativas inflacionárias, Regras de Taylor, Banco Central Brasileiro.

Classificação JEL: E17; E31; E52.

\section{INTRODUÇÃ̃O}

Um dos temas mais intrigantes e complexos na área de política monetária é o que trata da sistemática ou estratégia de ajustes no instrumento de política usado pelos Bancos Centrais no mundo. Em geral, as autoridades monetárias modernas fazem uso de uma taxa de juros de curtíssimo prazo como instrumento de política monetária, seja para gerar um efeito desejado

Professor Adjunto da UFES e Doutorado em Economia pela UFRJ. E-mail: ramalhete.s@gmail.com 
sobre o nível de atividade econômica, seja para controlar o processo inflacionário, de acordo com as normas estabelecidas.

No Brasil, o Banco Central (BCB) faz uso da taxa Selic, uma taxa interfinanceira de curtíssimo prazo, como ferramenta principal para que a inflação anual fique no entorno da meta de inflação. No entanto, quais são as variáveis que influenciam sistematicamente na tomada de decisão do BCB no que diz respeito ao valor fixado como meta para a taxa Selic? Em outras palavras, quais são as variáveis explicativas na determinação da regra de política monetária no Brasil?

Trata-se de questão de grande importância tanto no meio acadêmico quanto na prática do mercado financeiro. Naquele porque o meio científico em Economia possui interesse em compreender como as decisões do Banco Central estão sendo determinadas, e desta forma lançar luz sobre assuntos de relevância para a academia em particular, e para a sociedade em geral, tais como os assuntos relativos às intenções e aos objetivos de política monetária e à capacidade que a autoridade possui para alcançá-los e entregá-los ao público. Já no meio profissional, ligado ao mercado financeiro em especial, sabe-se que os movimentos das taxas interfinanceiras de juros têm grande impacto nas expectativas do mercado e na formação temporal de preços importantes como os das ações, títulos de renda fixa e os das moedas estrangeiras. Portanto, conhecer quais são os argumentos que afetam a formação da taxa básica de juros representa uma peça especial nas estratégias financeiras de empresas ligadas ao setor financeiro e, cada vez mais verdade, ao produtivo.

O presente trabalho estuda a formação da meta de taxa Selic no Brasil, no período de janeiro de 2005 a junho de 2010. Parte-se do referencial teórico das regras de política monetária, iniciado a partir dos anos $90 \mathrm{com}$ o trabalho pioneiro de John Taylor (1993), e desenvolvido com vasta gama de pesquisa nos últimos anos, a exemplo de Ball (1999), Blinder (2006), Woodford (2003) e Galí \& Gertler (2007). Conforme essa literatura, verifica-se que as regras de política monetária são flexíveis e mudam ao longo do tempo ou de acordo com preferências específicas de cada país, porém, em geral, mostra-se que as taxas básicas de juros dependem da dinâmica observada de todas as variáveis que determinam a inflação ao longo do tempo. Esta parece ter sido uma das mensagens deixadas por Taylor (1993).

O estudo utiliza dados mensais de produção industrial, da inflação ao consumidor (IPCA), das expectativas de inflação para 12 meses à frente e 
da meta de taxa anual de juros Selic verificada em cada mês da série, todas as variáveis coletadas para as 66 observações do período. A metodologia de análise econométrica será detalhada ao longo do trabalho, mas grosso modo trata-se de estimativas para regressões estacionárias multivariadas simples (Mínimos Quadrados Ordinários - MQO), buscando-se verificar qual a melhor regra estimada, através de alguns critérios de informação.

Basicamente, os resultados da análise mostram que o $\mathrm{BCB}$, no período estudado, ajusta a meta de taxa Selic de forma bastante gradual, ou seja, há grande inércia no ajuste do instrumento de política monetária, tal como apontado pela literatura recente (Woodford, 2003). Adicionalmente, observa-se grande peso das mudanças nas expectativas de inflação sobre as decisões de política, e relativamente pouco peso das observações passadas e correntes sobre as mesmas decisões. Logo, parece haver teor preventivo ou forward-looking ${ }^{2}$ na regra de política do $\mathrm{BCB}$, como também sugere a literatura sobre o tema (Clarida, Galí \& Gertler, 1999). Além disto, verificou-se que a dinâmica do gap do produto tem pouco poder de explicação no fenômeno analisado - em conformidade com os resultados de Minela et. al. (2003) -, ou seja, a taxa Selic parece responder muito pouco à dinâmica da atividade econômica, contrariando a teoria sobre o assunto. No entanto, como será argumentado, isto pode ser causado pela composição da variável adotada como proxy do gap do PIB, não significando que o BCB de fato dê pouca atenção à dinâmica produtiva. Assim, as estimativas encontradas dão suporte a determinadas proposições da teoria, porém não podem ser interpretadas como o verdadeiro modus operandi do BCB.

Adicionalmente, o trabalho realiza um pequeno exercício VAR bivariado com a finalidade de verificar a dinâmica interdependente entre as expectativas de inflação e a inflação corrente. Verificou-se que as expectativas de inflação, fator determinante das decisões de política monetária, são determinadas com precedência temporal pela inflação observada, demonstrando um padrão adaptativo na formação das projeções inflacionárias no período amostral.

O mesmo não se pode dizer a respeito da relação inversa, ou seja, não foram encontradas evidências de que as expectativas de inflação tenham peso

O comportamento forward-looking de um banco central diz respeito a um processo em que as decisões de instrumento de política são tomadas com base em projeções para a dinâmica futura das variáveis consideradas relevantes. 
relevante na dinâmica da inflação corrente, o que, por sua vez, aponta para uma curva de Phillips backward-looking ${ }^{3}$ na economia brasileira recente.

\section{UMA BREVE PERSPECTIVA DO REFERENCIAL TEÓRICO}

A partir dos anos 90, estabeleceu-se uma forma convencional de regra de política monetária, conhecida na literatura mundial como Regra de Taylor (Taylor, 1993). Basicamente, trata-se de uma equação de resposta da taxa de juros de curtíssimo prazo, ou seja, uma regra de reação da taxa básica de juros face à dinâmica de variáveis consideradas relevantes para a estratégia dos Bancos Centrais. Grosso modo, o conjunto relevante de informações consiste de variáveis que indiquem a dinâmica inflacionária e de atividade econômica.

O modelo estrutural de uma economia sob regime de metas de inflação pode ser explicado na linha oferecida por Ball (1999), Svensson (1999) e Galí \& Gertler (2007). Inicialmente, tem-se uma curva IS dinâmica estocástica, em que o desvio do produto em relação ao produto potencial no período corrente $\left(\mathrm{y}_{\mathrm{t}}\right)$ é determinado pelo seu valor defasado $\left(\mathrm{y}_{\mathrm{t}-1}\right)$, pelo desvio da taxa básica de juros $\left(\mathrm{i}_{\mathrm{t}-1}\right)$ e por um componente estocástico, um choque de demanda agregada $\left(\varepsilon_{1 t}\right)$, com média zero e variância constante. Ademais, os parâmetros $b_{1}$ e $b_{2}$ são ambos positivos:

$$
\text { (1) } y_{t}=b_{1} y_{t-1}+b_{2} i_{t-1}+\varepsilon_{1 t}
$$

Tem-se, por sua vez, uma curva de Phillips dinâmica; o desvio da inflação em relação à meta inflacionária no período corrente $\left(\pi_{t}\right)$ depende de seu valor em t-1, do desvio defasado do produto e de um componente aleatório, um choque de oferta $\left(\varepsilon_{2 t}\right)$, com média zero e variância constante. Sendo $\mathrm{a}_{1}$ e $\mathrm{a}_{2}$ parâmetros positivos:

$$
\text { (2) } \pi_{t}=a_{1} \pi_{t-1}+a_{2} y_{t-1}+\varepsilon_{2 t}
$$

O Banco Central adota uma regra de política monetária tipo regra de Taylor, em que a taxa básica de juros é função dos níveis do desvio do pro-

Uma curva de Phillips backward-looking ocorre quando a taxa de inflação corrente está determinada pelos seus valores passados, assim como pelos valores passados da atividade econômica. 
duto e da inflação face a seus valores desejados, sendo $\mathrm{r}^{\mathrm{n}}$ a taxa real de juros de equilíbrio e $\Pi^{\mathrm{T}}$ a meta de inflação. Os parâmetros $\mathrm{c}_{1}$ e $\mathrm{c}_{2}$ são positivos e $\varepsilon_{3 \mathrm{t}}$ é um choque de inovação de política:

$$
\text { (3) } i_{t}=r^{n}+\Pi^{T}+c_{1} y_{t-1}+c_{2} \pi_{t-1}+\varepsilon_{3 t}
$$

A literatura demonstra que os Bancos Centrais possuem um comportamento inercial no que respeita ao ajuste da taxa básica de juros (Woodford, 2003; Blinder, 2006). Ou seja, ao definir a cada momento a nova taxa básica na economia, os policymakers dão peso aos valores passados dessa taxa. Isto seria uma maneira de suavização da política monetária, evitando-se movimentos abruptos e reversões freqüentes da taxa básica de juros. Assim, adiciona-se à equação (3) um componente de suavização ou inércia de juros:

$$
\text { (4) } i_{t}=\rho i_{t-1}+(1-\rho)\left[\left(r^{n}+\Pi^{T}\right)+c_{1} y_{t-1}+c_{2} \pi_{t-1}\right]+\varepsilon_{4 t}
$$

Onde $\rho(0 \leq \rho \leq 1)$ é o coeficiente de inércia, que dá o peso da taxa defasada na formação da taxa corrente, enquanto $\varepsilon_{4 \mathrm{t}}$ é o choque de inovação de política na regra inercial.

De forma alternativa, muitas variações da regra de Taylor (1993) são oferecidas e testadas pela literatura, as quais adotam especificações diferentes e/ou variáveis explicativas adicionais para a determinação dos ajustes de taxas básicas de juros. Alguns modelos possuem caráter forward-looking, ao dar à expectativa inflacionária um peso importante sobre a decisão das autoridades. Haldane \& Batini (1998), por exemplo, adotam uma regra de instrumento com o seguinte perfil:

$$
\text { (5) } r_{t}=\rho r_{t-1}+(1-\rho) r_{t}^{*}+\theta\left[E_{t} \Pi_{t+j}-\Pi_{t}^{*}\right]+\varphi y_{t-1}
$$

Em que $r_{t}=\left[i_{t}-E_{t} \Pi_{t+1}\right]$, é a taxa real de juros esperada, qual seja, a diferença entre a taxa nominal de juros $\left(i_{t}\right)$ praticada em t e a inflação esperada em $t\left(E_{t} \Pi_{t+1}\right)$ para $t+1$. A taxa real de juros de equilíbrio é dada por $r_{t}^{*}$, a meta de inflação é dada por $\Pi_{t}^{*}$ e os parâmetros $\rho, \theta$ e $\varphi$ são positivos. Clarida, Galí \& Gertler (1999), dentre outros, adotam principalmente regras forward-looking, ao invés de bacward-looking, como uma boa descrição do comportamento das taxas básicas de juros em economias com estratégia implícita ou explícita - de estabilidade de preços. 
Em particular no que se refere à economia brasileira, Minella et. al. (2003) fizeram uso do desvio da inflação esperada em relação à meta de inflação como componente determinante do instrumento de política, a partir de um estudo de regressões no período 1999/06 - 2002/06. Ademais, foi testada uma equação com duas defasagens da taxa básica de juros, com o intuito de eliminar o problema da autocorrelação serial, observada no modelo com apenas uma defasagem da Selic. Os resultados em Minella (op. cit.), em resumo, foram os seguintes: a) foi observada elevada inércia da política monetária: variando entre $0.7-0.8$ ou mais o coeficiente $\rho$; b) o coeficiente do gap do produto não obteve significância estatística, em geral, ou apresentou sinal não consistente com a teoria em alguns casos; c) os autores também testaram o modelo com inclusão da variação da taxa de câmbio como termo explicativo da equação, porém o seu coeficiente não foi significante estatisticamente; d) o coeficiente do desvio de expectativa inflacionária $(\theta)$, em geral, mostrou magnitude maior que um e significância ao nível de $1 \%$.

Este último resultado também está presente no trabalho desenvolvido por Holland (2005), assim como no de Barbosa \& Soares (2006), para a economia brasileira, denotando uma postura contra-cíclica por parte do $\mathrm{BCB}$. Como será visto, o presente trabalho também encontra evidências, dentre outras, de que o BCB possui comportamento preventivo e agressivo no controle da inflação, ajustando a meta Selic mais do que proporcionalmente - coeficiente maior que 1 - às mudanças das expectativas de inflação.

\section{METODOLOGIA}

Busca-se testar a aderência estatística de regras de política monetária, tipo as equações (4 e 5), para a economia brasileira recente (2005/012010/06). Este período é escolhido porque apresenta uma estabilidade da meta de inflação em $4.5 \%$ para o IPCA-IBGE; desta forma, contorna-se o problema das mudanças na meta inflacionária presente em Minella et. al. (2003). As variáveis utilizadas nos testes serão as seguintes:

$\mathrm{i}_{\mathrm{t}}=\mathrm{a}$ meta de taxa básica de juros (Selic) fixada pelo Banco Central do Brasil para cada mês da série temporal, vigente entre os dias 06 a 10 do respectivo mês (fonte: www.bcb.gov.br); 
$\pi_{\mathrm{t}}=\left(\mathrm{ipca}_{\mathrm{t}} / \mathrm{ipca}_{\mathrm{t}-1}\right)=\mathrm{o}$ desvio da inflação acumulada nos últimos 12 meses em relação à inflação acumulada até o mês t-1, medidas pelo Índice de Preços ao Consumidor Amplo (IPCA/fonte: www.ibge.gov.br);

$\pi_{t}^{\mathrm{e}}=\mathrm{E}_{\mathrm{t}}\left[\pi_{\mathrm{t}+12}\right] / \mathrm{E}_{\mathrm{t}-1}\left[\pi_{\mathrm{t}+11}\right]=$ desvio da expectativa de inflação acumulada para 12 meses à frente, formada no mês $t$, em relação à mesma expectativa formada no mês t-1. Os dados de expectativas inflacionárias são disponibilizados pelo Sistema Gerador de Séries do BC. (fonte: www.bcb.gov.br);

$\mathrm{y}_{\mathrm{t}}=$ desvio do produto industrial da economia brasileira em relação ao produto industrial potencial. Utilizaram-se os dados do índice dessazonalizado de produção mensal da indústria geral (fonte: www.ipeadata.gov. br) como proxy do PIB, já que este último não está disponível diretamente em periodicidade mensal. Para o cálculo do desvio do produto industrial face ao produto potencial foi utilizado um filtro estatístico, o filtro de $\mathrm{Ho}$ drick-Prescott $^{4}(H P)$, que caracteriza uma série temporal como a soma de um componente de tendência variável suavizada com um componente de ciclo. Isto se fez necessário porque, como se sabe, não há dados diretos para o valor do produto potencial industrial. Logo, a variável y é o componente de ciclo extraído pelo filtro HP, com base no índice utilizado. Como se pode observar no Gráfico 4, obtêm-se algumas observações de valor negativo para o gap do produto, o que é natural quando o produto efetivo está abaixo do valor potencial (tendência). Essas observações negativas impedem o uso dos valores em logaritmos, por outro lado; O Anexo 1 apresenta o comportamento gráfico das séries utilizadas.

O trabalho parte dos testes convencionais de raiz unitária e estacionariedade para as séries adotadas. Como é conhecido, utilizar inadvertidamente séries temporais não-estacionárias em análises de regressão pode conduzir o pesquisador a resultados espúrios (Granger \& Newbold, 1974).

A estacionariedade (não-existência de raiz unitária) de uma série ocorre quando: a) a série apresenta média constante $-\mathrm{E}\left(\mathrm{x}_{\mathrm{t}}\right)=\mu$; b) a série apresenta variância constante $\left.-\operatorname{Var}\left(x_{t}\right)=\sigma^{2} ; c\right)$ a covariância entre os valores de uma série depender tão apenas da distância entre os tempos em que tais valores

Hodrick \& Prescott (1997) 
são observados, e nunca dos tempos reais em que são observados - $\operatorname{COV}\left(\mathrm{x}_{\mathrm{t}}\right.$, $\left.\mathrm{x}_{\mathrm{t}-1}\right)=\operatorname{COV}\left(\mathrm{x}_{\mathrm{t}}, \mathrm{x}_{\mathrm{t}+1}\right)$. Assim, a estacionariedade requer que a natureza de qualquer correlação entre termos adjacentes de uma variável estocástica seja a mesma ao longo de todos os períodos (Wooldridge, 2009).

Será realizado o teste de raiz unitária de Dickey-Fuller Aumentado (DFA) - Dickey \& Fuller (1979), Dickey \& Fuller (1981), Fuller (1976) -, além do teste de estacionariedade de Kwiatkowski-Phillips-Schmidt-Shin $(\mathrm{KPSS})^{5}$. Caso as séries não sejam estacionárias, porém integradas de mesma ordem, proceder-se-á aos testes de cointegração, analisando-se a possibilidade de estacionariedade dos resíduos entre as variáveis, por meio da metodologia de Enders (2004). O teste DFA testa raiz unitária no contexto do seguinte modelo, sendo x uma variável estocástica qualquer:

$$
\text { (6) } \Delta \mathrm{x}_{\mathrm{t}}=\mathrm{a}_{0}+\mathrm{a}_{1} \mathrm{t}+\gamma \mathrm{x}_{\mathrm{t}-1}+\left(\beta_{1} \Delta \mathrm{x}_{\mathrm{t}-1}+\ldots+\beta_{\mathrm{j}} \Delta \mathrm{x}_{\mathrm{t}-\mathrm{j}}\right)+v_{\mathrm{t}}
$$

A variável x é estacionária quando rejeita-se a hipótese nula de que $\gamma=0$ - correspondente ao caso em que $\alpha=1$ numa equação simples $x_{t}=$ $\alpha \mathrm{x}_{\mathrm{t}-1}+\mathrm{e}_{\mathrm{t}}$. . Deve-se frisar que (6) é o modelo ampliado, com constante $\left(\mathrm{a}_{0}\right)$ e tendência $\left(a_{1} t\right)$. No caso destes componentes não serem significantes estatisticamente, deve-se testar a hipótese $\gamma=0$ sem os mesmos na equação. O componente somatória $\left(\beta_{1} \Delta \mathrm{x}_{\mathrm{t}-1}+\ldots+\beta_{\mathrm{j}} \Delta \mathrm{x}_{\mathrm{t}-\mathrm{j}}\right)$ tem como finalidade permitir um processo ARMA (Auto-regressive Moving Average) no termo de erro (Box \& Jenkins, 1976; Maddala, 1992).

A existência de estacionariedade dos resíduos, entre variáveis integradas de mesma ordem, permite estabelecer uma relação de equilíbrio (no sentido estatístico) de longo prazo entre as mesmas. Logo, procedimentos de regressão relativamente simples podem dizer alguma coisa importante a respeito das sensibilidades de uma variável face às outras. No entanto, no caso das variáveis utilizadas serem estacionárias (integradas de ordem zero $\mathrm{I}(0)$ ), proceder-se-á diretamente ao uso de regressões múltiplas por meio de mínimos quadrados ordinários (MQO), a fim de que se verifique tão somente a aderência de regras de resposta do $\mathrm{BC}$ para a economia brasileira no período estudado.

Para detalhes sobre as propriedades estatísticas do KPSS, conferir Kwiatkowski et. al. ( I 992). 


\section{RESULTADOS E ANÁLISE}

\subsection{Testes de raiz unitária e estacionariedade}

Pelo exercício Dickey-Fuller Aumentado, chegou-se aos seguintes valores para os testes I (com tendência e constante), II (com constante) e III (sem tendência e sem constante), na Tabela 1. Por sua vez, o teste de estacionariedade de Kwiatkowski-Phillips-Schmidt-Shin (KPSS) apresentou os resultados da Tabela 2 .

Tabela 1 - Teste de raiz unitária Dickey-Fuller Aumentado - estatísticas $\tau$

\begin{tabular}{|c|c|c|c|}
\hline Variável em nível & Tendência e constante & Constante & $\begin{array}{c}\text { Sem tendência e } \\
\text { sem constante }\end{array}$ \\
\hline $\mathbf{i}$ (I) & $-3.638^{* *}$ & -2.919 & -1.796 \\
\hline$\pi$ (II) & -4.427 & $-4.418^{* * *}$ & -0.312 \\
\hline$\pi^{\mathrm{e}}$ (II) & -6.529 & $-6.350^{* * *}$ & 0.11 \\
\hline $\mathbf{y}$ (III) & -2.571 & -2.594 & $-2.618^{* * *}$ \\
\hline
\end{tabular}

(***) Rejeita a hipótese nula ao nível de $1 \%$; (**) rejeita a hipótese nula ao nível de 5\%; (I) constante e tendência significantes a $5 \%$; (II) apenas constante significante a $5 \%$; (III) constante e tendência não significantes a $5 \%$. Fonte: elaboração própria a partir das estatísticas do software econométrico utilizado.

Tabela 2 - Teste de estacionariedade Kwiatkowski-Phillips-Schmidt-Shin (KPSS)

\begin{tabular}{|c|c|c|}
\hline Variável em nível & Tendência e constante & Constante \\
\hline $\mathbf{i}$ (I) & $0.133390^{\star * *}$ & 0.866954 \\
\hline$\pi$ (II) & 0.120751 & $0.227999^{\star * *}$ \\
\hline$\pi^{\mathrm{e}}$ (II) & 0.089182 & $0.411008^{\star * *}$ \\
\hline $\mathbf{y}$ (III) & 0.070984 & $0.070984^{* * *}$ \\
\hline
\end{tabular}

$(* * *)$ Não rejeita a hipótese nula de estacionariedade ao nível de $1 \%$, nem ao nível de 5\%; (I) constante e tendência significantes a 5\%; (II) apenas constante significante a 5\%; Fonte: elaboração própria a partir das estatísticas do software econométrico utilizado.

Como todas as variáveis são consideradas estacionárias, com base nos testes DFA e KPSS realizados, utiliza-se diretamente Ordinary Least Squares (OLS) (mínimos quadrados ordinários); tal procedimento - uso de OLS quando da estacionariedade em nível das variáveis - está respaldado por, dentre outros, Hill, Griffiths \& Judge (1998). 


\subsection{Regras de política monetária estimadas: com a meta de taxa Selic em nível}

Foram estimadas 06 regras ou modelos de resposta do BCB para o período analisado. As especificações dessas regras são as seguintes:

Modelo 1: $\mathrm{i}_{\mathrm{t}}=\delta_{0}+\delta_{1} \mathrm{i}_{\mathrm{t}-1}+\delta_{2} \mathrm{i}_{\mathrm{t}-2}+\delta_{3} \mathrm{y}_{\mathrm{t}}+\delta_{4} \mathrm{y}_{\mathrm{t}-1}+\delta_{5} \pi_{\mathrm{t}}+\delta_{6} \pi_{\mathrm{t}-1}+\delta_{7} \pi_{\mathrm{t}}^{\mathrm{e}}+\delta_{8} \pi_{\mathrm{t}-1}^{\mathrm{e}}+\varepsilon_{1 \mathrm{t}}$ Modelo 2: $\mathrm{i}_{\mathrm{t}}=\delta_{0}+\delta_{1} \mathrm{i}_{\mathrm{t}-1}+\delta_{2} \mathrm{i}_{\mathrm{t}-2}+\delta_{3} \mathrm{y}_{\mathrm{t}-1}+\delta_{4} \pi_{\mathrm{t}-1}^{\mathrm{e}}+\varepsilon_{2 \mathrm{t}}$

Modelo 3: $\mathrm{i}_{\mathrm{t}}=\delta_{0}+\delta_{1} \mathrm{i}_{\mathrm{t}-1}+\delta_{2} \mathrm{i}_{\mathrm{t}-2}+\delta_{3} \mathrm{y}_{\mathrm{t}}+\delta_{4} \pi_{\mathrm{t}-1}^{\mathrm{e}}+\varepsilon_{3 \mathrm{t}}$

Modelo 4: $\mathrm{i}_{\mathrm{t}}=\delta_{0}+\delta_{1} \mathrm{i}_{\mathrm{t}-1}+\delta_{2} \mathrm{y}_{\mathrm{t}-1}+\delta_{3} \pi_{\mathrm{t}-1}^{\mathrm{e}}+\varepsilon_{4 \mathrm{t}}$

Modelo 5: $\mathrm{i}_{\mathrm{t}}=\delta_{0}+\delta_{1} \mathrm{i}_{\mathrm{t}-1}+\delta_{2} \mathrm{y}_{\mathrm{t}-1}+\delta_{3} \pi_{\mathrm{t}-1}+\varepsilon_{5 \mathrm{t}}$

Modelo 6: $\mathrm{i}_{\mathrm{t}}=\delta_{0}+\delta_{1} \mathrm{i}_{\mathrm{t}-1}+\delta_{2} \mathrm{y}_{\mathrm{t}-1}+\delta_{3} \pi_{\mathrm{t}-1}+\delta_{4} \pi_{\mathrm{t}-1}^{\mathrm{e}}+\varepsilon_{6 \mathrm{t}}$

Verifica-se que o Modelo 1 é o mais geral, incluindo como variáveis explicativas duas defasagens da taxa de juros $\left(\mathrm{i}_{\mathrm{t}-1}\right.$ e $\left.\mathrm{i}_{\mathrm{t}-2}\right)$, o gap corrente e passado do produto, o desvio inflacionário corrente e passado e o desvio corrente e passado das expectativas de inflação; os demais modelos são mais parcimoniosos, face ao Modelo 01, já que incluem um número menor de variáveis explicativas, e suas especificações são inteligíveis para o leitor. Todos os modelos foram estimados com constantes.

Essas especificações, de certa forma, foram escolhidas arbitrariamente. Um número maior de modelos poderia ter sido testado, porém tal exercício daria maior exaustão ao trabalho e não chegaria necessariamente a modelos com melhor ajuste aos dados. No entanto, esse experimento pode ser ampliado em futuros trabalhos.

A Tabela 3 apresenta os valores encontrados para os coeficientes estimados dos regressores em cada modelo e para os desvios-padrão (valores entre parênteses); além disto, para cada modelo, são listadas as seguintes estatísticas: $\mathrm{R}^{2}$ ajust. - o coeficiente de determinação ajustado; AIC - o critério de informação de Akaike; SIC - o critério de informação de Schwarz; HQ - o critério de informação Hannan-Quinn; LM - a estatística (p-valor) do teste de auto-correlação dos resíduos de Breusch-Godfrey ${ }^{6}$. A significância

Todos os modelos testados apresentaram auto-correlação dos resíduos, pela estatística LM (p-valor), ao nível de 10\% de significância. Logo, aplicou-se o método de correção de Newey-West em todos os modelos testados. As estatísticas reportadas, portanto, dizem respeito às regressões de mínimos quadrados corrigidas do problema de 
estatística dos coeficientes estimados é indicada por (***), quando ao nível de $1 \%$, e $(* *)$, quando a $5 \%$.

Todos os modelos testados possuem coeficiente de determinação ajustado ( $\mathrm{R}^{2}$ ajust.) acima de 0.98 , indicando elevado ajuste das regras de política estimadas, ou seja, aproximadamente $98 \%$ das variações na meta de taxa Selic, no período de amostra, são explicados a partir das variáveis exógenas adotadas em cada especificação.

Observa-se que a primeira defasagem da taxa Selic é bastante significativa em todos os modelos testados, com elevado poder de explicação - coeficiente em torno de 1 . Nas regras estimadas com duas defasagens, o valor do coeficiente da primeira defasagem aumenta, porém o valor líquido dos coeficientes da primeira e segunda defasagem continua em torno da unidade. Este resultado aponta para uma elevada inércia da política monetária no país, ao longo do período recente, e corrobora a tese de que a suavização (smoothing) dos ajustes de taxa de juros, por parte dos bancos centrais, pode ser vista como um fato estilizado nas economias modernas (Woodford, 2003; Blinder, 2006; Galí \& Gertler, 2007).

Por sua vez, em todos os modelos em que aparece, a primeira defasagem das expectativas de inflação é a variável com maior poder de explicação sobre as decisões de taxa de juros do $\mathrm{BCB}$ - variando o coeficiente entre 1.5 a 2.2. Apenas no Modelo 6 esta variável não possui significância estatística ao nível de $5 \%$.

Pode-se dizer que as expectativas inflacionárias 12 meses à frente são uma informação de grande relevância na formação da estrutura de juros no Brasil recente. No entanto, o mesmo não ocorre com as expectativas inflacionárias correntes, as quais não apresentam significância estatística (Modelo 1 e outros modelos testados) ${ }^{7}$. Grosso modo, tomando como base a significância estatística e o elevado coeficiente encontrado para a primeira defasagem do desvio das expectativas de inflação como determinante das decisões de meta de taxa Selic, pode-se concordar com as palavras de Minella et. al. (2003, p. 13), embora os últimos a partir de uma outra amostra, de que "we can conclude that the Central Bank has been reacting strongly

auto-correlação dos resíduos.

Este resultado pode ser interpretado pela existência de uma inércia na compreensão e avaliação das expectativas que estão sendo divulgadas contemporaneamente, de maneira que as decisões em t levam em conta as expectativas em $\mathrm{t}-\mathrm{I}$, as quais já foram "digeridas" pelo BCB. 
to expected inflation. It conducts monetary policy on a forward-looking basis, and responds to inflationary pressures".

Já o desvio corrente e passado da inflação, embora com coeficientes expressivos, não demonstrou confiança estatística, como se observa nos Modelos 1, 5 e 6. E o gap do produto, apesar de significativo estatisticamente na maioria dos casos, apresenta pouco poder de explicação na regra de política do $\mathrm{BCB}$ - com valor do coeficiente variando entre 0.023 a 0.038 .

Algumas observações devem ser feitas a respeito do gap do produto e de sua baixa capacidade explicativa nas regras testadas. Como se disse na metodologia do trabalho, utilizou-se a série de produção industrial mensal da economia brasileira, divulgada pelo IBGE, como proxy do gap do PIB. Ademais, a variável $y$ nas regras é extraída pelo filtro HP, ou seja, o gap do produto usado nos modelos deste trabalho é formado pelo componente de ciclo extraído do filtro estatístico.

Assim, pode-se argumentar no sentido de que esta variável não representa com exatidão as informações de atividade econômica usadas pelo $\mathrm{BCB}$ ao decidir sobre a meta de taxa Selic, o que estaria sendo refletido nos reduzidos valores captados pelos coeficientes nos modelos testados. Não obstante, o procedimento aqui adotado - o uso da variável produção industrial e do filtro HP - é amplamente implementado (Conferir Bogdanski, Tombini \& Werlang (2000) e Minella et. al. (2003)).

Os critérios utilizados para a escolha dos modelos que melhor se ajustam aos dados foram os de Akaike (AIC), Schwarz (SIC) e Hannan-Quinn (HQ). Estatísticas menores para esses testes indicam melhor ajuste do respectivo modelo aos dados reais, em relação aos demais modelos. Como se verifica pela Tabela 3, o modelo que apresentou menores valores para os critérios AIC, SIC e HQ - indicando melhor aderência aos dados - foi o Modelo 2. Concomitantemente, este foi o modelo que apresentou maior valor para o coeficiente de determinação ajustado $\left(R^{2}\right.$ ajust.). A regra de política é a seguinte:

$\mathrm{i}_{\mathrm{t}}=-2.010727+1.207118 \mathrm{i}_{\mathrm{t}-1}-0.207723 \mathrm{i}_{\mathrm{t}-2}+0.029178 \mathrm{y}_{\mathrm{t}-1}+1.91482 \pi_{\mathrm{t}-1}^{\mathrm{e}}+\varepsilon_{2 \mathrm{t}}$

Grosso modo, os sinais dos coeficientes $\left(\delta_{0}, \delta_{1}, \delta_{2}, \delta_{3}\right.$ e $\left.\delta_{4}\right)$ estão como o esperado pela teoria. A política monetária age contra-ciclicamente face ao gap do produto $\left(\delta_{3}>0\right)$, embora essa resposta seja tecnicamente nula; no entanto, a resposta face a uma mudança no desvio das expectativas de inflação 
indica alto teor contra-cíclico, uma vez que $\delta_{4}>1$, ou seja, diante de uma elevação das expectativas de inflação, a meta de taxa Selic aumenta mais do que proporcionalmente. Isto implica aumento da taxa real de juros esperada na economia, em consonância com o princípio de Taylor ${ }^{8}$. A constatação de um $\delta_{4}>1$ é consistente também com uma série de trabalhos empíricos aplicados ao caso Brasileiro, sob o regime de metas para inflação, dentre os quais Minella et. al. (2003), Holland (2005) e Barbosa \& Soares (2006).

Tabela 3 - Modelos multivariados estimados para o nível da taxa de juros: coeficientes estimados para os regressores, desvios-padrão e estatísticas de ajuste e previsão - regressões corrigidas da auto-correlação dos resíduos pelo método de Newey-West

\begin{tabular}{|c|c|c|c|c|c|c|}
\hline & Modelo 1 & Modelo 2 & Modelo 3 & Modelo 4 & Modelo 5 & Modelo 6 \\
\hline constante & $\begin{array}{c}-1.877542 \\
(-1.580625)\end{array}$ & $\begin{array}{c}-2.010727^{* *} \\
(0.841051)\end{array}$ & $\begin{array}{c}-2.403955^{* *} \\
(1.023601)\end{array}$ & $\begin{array}{c}-1.914102^{* *} \\
(0.879057)\end{array}$ & $\begin{array}{l}-1.303798 \\
(1.182822) \\
\end{array}$ & $\begin{array}{c}-2.543171 \\
(1.277649) \\
\end{array}$ \\
\hline $\mathbf{i}_{\mathrm{t}-1}$ & $\begin{array}{c}1.192677 * * * \\
(0.163129)\end{array}$ & $\begin{array}{c}1.207118 * * * \\
(0.154345)\end{array}$ & $\begin{array}{c}1.260665 * * * \\
(0.112840)\end{array}$ & $\begin{array}{c}1.005362 * * * \\
(0.019177)\end{array}$ & $\begin{array}{c}1.003605 * * * \\
(0.019747)\end{array}$ & $\begin{array}{c}1.007989^{* * *} \\
(0.019776)\end{array}$ \\
\hline $\mathbf{i}_{\mathrm{t}-2}$ & $\begin{array}{l}-0.192486 \\
(0.162228) \\
\end{array}$ & $\begin{array}{c}-0.207723 \\
(0.152753) \\
\end{array}$ & $\begin{array}{c}-0.259037 * * \\
(0.115755)\end{array}$ & $\begin{array}{l}- \\
-\end{array}$ & $\begin{array}{l}- \\
-\end{array}$ & $\begin{array}{l}- \\
-\end{array}$ \\
\hline$y_{t}$ & $\begin{array}{l}-0.001835 \\
(0.007766) \\
\end{array}$ & - & $\begin{array}{c}0.023023 \\
(0.013090) \\
\end{array}$ & - & $\begin{array}{l}- \\
-\end{array}$ & $\begin{array}{l}- \\
-\end{array}$ \\
\hline $\mathbf{y}_{\mathrm{t}-1}$ & $\begin{array}{c}0.030541^{* *} \\
(0.013861)\end{array}$ & $\begin{array}{c}0.029178 * * * \\
(0.011350)\end{array}$ & $\begin{array}{l}- \\
-\end{array}$ & $\begin{array}{c}0.038009 * * * \\
(0.010372)\end{array}$ & $\begin{array}{c}0.038297 * * * \\
(0.010898)\end{array}$ & $\begin{array}{c}0.035343^{* * *} \\
(0.010615)\end{array}$ \\
\hline$\pi_{t}$ & $\begin{array}{c}0.778839 \\
(0.802883)\end{array}$ & - & - & $\begin{array}{l}- \\
-\end{array}$ & - & $\begin{array}{l}- \\
-\end{array}$ \\
\hline$\pi_{\mathrm{t}-1}$ & $\begin{array}{c}0.668314 \\
(1.008423)\end{array}$ & $\begin{array}{l}- \\
-\end{array}$ & $\begin{array}{l}- \\
-\end{array}$ & $\begin{array}{l}- \\
-\end{array}$ & $\begin{array}{c}1.134988 \\
(1.088104) \\
\end{array}$ & $\begin{array}{c}0.809099 \\
(1.040037) \\
\end{array}$ \\
\hline$\pi_{t}^{e}$ & $\begin{array}{l}-1.552016 \\
(0.966811) \\
\end{array}$ & $\begin{array}{l}- \\
- \\
\end{array}$ & $\begin{array}{l}- \\
- \\
\end{array}$ & - & $\begin{array}{l}- \\
-\end{array}$ & $\begin{array}{l}- \\
-\end{array}$ \\
\hline$\pi_{t-1}^{e}$ & $\begin{array}{l}1.878045^{* *} \\
(0.781797) \\
\end{array}$ & $\begin{array}{c}1.914820 * * * \\
(0.800185)\end{array}$ & $\begin{array}{c}2.283117^{* *} \\
(0.969713) \\
\end{array}$ & $\begin{array}{l}1.721361^{* *} \\
(0.839399) \\
\end{array}$ & - & $\begin{array}{c}1.508513 \\
(0.875983) \\
\end{array}$ \\
\hline$R^{2}$ ajust. & 0.989377 & 0.989580 & 0.989087 & 0.988961 & 0.988711 & 0.988914 \\
\hline AIC & 0.899927 & 0.825801 & 0.872063 & 0.884158 & 0.906520 & 0.902660 \\
\hline SIC & 1.203520 & 0.994463 & 1.040726 & 1.017967 & 1.040328 & 1.069920 \\
\hline HQ & 1.019527 & 0.892245 & 0.938508 & 0.936954 & 0.959316 & 0.968655 \\
\hline LM & $0.083(6 \operatorname{lgs})$ & 0.033 (5 lgs) & 0.016 (1 lag) & 0.037 (6 lgs) & 0.037 (3 lgs) & $.061(6 \operatorname{lgs})$ \\
\hline
\end{tabular}

Fonte: elaboração própria a partir das estatísticas do software econométrico utilizado.

A idéia de que o coeficiente de resposta da taxa nominal de juros, diante de uma elevação da inflação, deve ter magnitude maior que I, a fim de que haja elevação da taxa real de juros. 
A inércia da política monetária, contudo, apresenta teor extremo, visto que $\delta_{1}>1$. Embora $\delta_{1}+\delta_{2}<1, \delta_{2}$ não é significativo a $5 \%$. Neste caso, pelo componente de inércia, a meta Selic apresentaria, coeteris paribus, um aumento ao longo do tempo; o termo de constante $\delta_{0}<0$, por sua vez, contribui para a tendência observada de queda da Selic no período amostral, dado que $\delta_{0}+\delta_{1}<0$.

\subsection{Regras para a taxa Selic em primeira diferença}

Adicionalmente, realizam-se testes para a resposta da variação da taxa de juros $\left(\Delta \mathrm{i}_{\mathrm{t}}=\mathrm{i}_{\mathrm{t}}-\mathrm{i}_{\mathrm{t}-1}\right)$ diante das mesmas variáveis, utilizando-se as mesmas especificações da Tabela 3. Este exercício é importante porque poder captar efeitos das mudanças nas variáveis explicativas sobre a primeira diferença da taxa de juros Selic, complementando o estudo daqueles mesmos efeitos sobre a formação do nível dessa taxa, a exemplo do experimento em Minella et. al. (2003). Logo, as especificações são as seguintes:

Modelo i: $\Delta \mathrm{i}_{\mathrm{t}}=\delta_{0}+\delta_{1} \Delta \mathrm{i}_{\mathrm{t}-1}+\delta_{2} \Delta \mathrm{i}_{\mathrm{t}-2}+\delta_{3} \mathrm{y}_{\mathrm{t}}+\delta_{4} \mathrm{y}_{\mathrm{t}-1}+\delta_{5} \pi_{\mathrm{t}}+\delta_{6} \pi_{\mathrm{t}-1}+\delta_{7} \pi_{\mathrm{t}}^{\mathrm{e}}+$ $\delta_{8} \pi^{\mathrm{e}}{ }_{\mathrm{t}-1}+\varepsilon_{1 \mathrm{t}}$

Modelo ii: $\Delta \mathrm{i}_{\mathrm{t}}=\delta_{0}+\delta_{1} \Delta \mathrm{i}_{\mathrm{t}-1}+\delta_{2} \Delta \mathrm{i}_{\mathrm{t}-2}+\delta_{3} \mathrm{y}_{\mathrm{t}-1}+\delta_{4} \pi_{\mathrm{t}-1}^{\mathrm{e}}+\varepsilon_{2 \mathrm{t}}$

Modelo iii: $\Delta \mathrm{i}_{\mathrm{t}}=\delta_{0}+\delta_{1} \Delta \mathrm{i}_{\mathrm{t}-1}+\delta_{2} \Delta \mathrm{i}_{\mathrm{t}-2}+\delta_{3} \mathrm{y}_{\mathrm{t}}+\delta_{4} \pi_{\mathrm{t}-1}^{\mathrm{e}}+\varepsilon_{3 \mathrm{t}}$

Modelo iv: $\Delta \mathrm{i}_{\mathrm{t}}=\delta_{0}+\delta_{1} \Delta \mathrm{i}_{\mathrm{t}-1}+\delta_{2} \mathrm{y}_{\mathrm{t}-1}+\delta_{3} \pi_{\mathrm{t}-1}^{\mathrm{e}}+\varepsilon_{4 \mathrm{t}}$

Modelo v: $\Delta \mathrm{i}_{\mathrm{t}}=\delta_{0}+\delta_{1} \Delta \mathrm{i}_{\mathrm{t}-1}+\delta_{2} \mathrm{y}_{\mathrm{t}-1}+\delta_{3} \pi_{\mathrm{t}-1}+\varepsilon_{5 \mathrm{t}}$

Modelo vi: $\Delta \mathrm{i}_{\mathrm{t}}=\delta_{0}+\delta_{1} \Delta \mathrm{i}_{\mathrm{t}-1}+\delta_{2} \mathrm{y}_{\mathrm{t}-1}+\delta_{3} \pi_{\mathrm{t}-1}+\delta_{4} \pi_{\mathrm{t}-1}^{\mathrm{e}}+\varepsilon_{6 \mathrm{t}}$

O Modelo i, o mais geral, coloca a variação corrente da meta de taxa Selic $\left(\Delta \mathrm{i}_{\mathrm{t}}\right)$ como explicada por essa variação em $\mathrm{t}-1\left(\Delta \mathrm{i}_{\mathrm{t}-1}\right)$ e $\mathrm{t}-2\left(\Delta \mathrm{i}_{\mathrm{t}-2}\right)$, pelo gap do produto corrente e passado, pelo desvio da inflação corrente e passado e pelo desvio das expectativas inflacionárias também nos períodos $\mathrm{t}$ e t-1. Os demais modelos ii a vi são mais parcimoniosos, com diferentes especificações a partir das variáveis no Modelo i. Todas as regressões, a exemplo dos Modelos 1 a 6, apresentaram auto-correlação dos resíduos (estatística LM). Logo, aplicou-se o método de Newey-West para a correção do problema em todos os modelos. A Tabela 4 mostra os valores encontrados para as especificações $i$ a vi. As estatísticas computadas são as mesmas para as regras de política em nível. 
A regra de variação da taxa de juros que melhor se ajusta aos dados é a regra ii, com base nas estatísticas de Akaike (AIC), Schwarz (SIC) e HannanQuinn (HQ), assim como pelo coeficiente de determinação ajustado ( $\mathrm{R}^{2}$ ajust.):

$\Delta \mathrm{i}_{\mathrm{t}}=-1.986757+0.082271 \Delta \mathrm{i}_{\mathrm{t}-1}+0.257638 \Delta \mathrm{i}_{\mathrm{t}-2}+0.027152 \mathrm{y}_{\mathrm{t}-1}+$ $1.891580 \pi_{\mathrm{t}-1}^{\mathrm{e}}+\varepsilon_{2 \mathrm{t}}$

Tabela 4 - Modelos estimados para a variação da taxa de juros: coeficientes estimados para os regressores, desvios-padrão e estatísticas de ajuste e previsão - regressões corrigidas da auto-correlação dos resíduos pelo método de Newey-West

\begin{tabular}{|c|c|c|c|c|c|c|}
\hline & Modelo i & Modelo ii & Modelo iii & Modelo iv & Modelo v & Modelo vi \\
\hline constante & $\begin{array}{c}-1.705499 \\
(1.355782)\end{array}$ & $\begin{array}{c}-1.986757 * * \\
(0.921817)\end{array}$ & $\begin{array}{c}-2.140816^{* *} \\
(0.984824)\end{array}$ & $\begin{array}{c}-2.030306^{* *} \\
(0.842953)\end{array}$ & $\begin{array}{c}-1.122898 \\
(0.908327)\end{array}$ & $\begin{array}{c}-2.379510 * * \\
(1.131313)\end{array}$ \\
\hline$\overline{\Delta \mathbf{i}_{\mathrm{t}-1}}$ & $\begin{array}{c}0.085010 \\
(0.117017)\end{array}$ & $\begin{array}{c}0.082271 \\
(0.104457)\end{array}$ & $\begin{array}{c}0.109078 \\
(0.098295)\end{array}$ & $\begin{array}{c}0.207170 \\
(0.152663)\end{array}$ & $\begin{array}{c}0.171996 \\
(0.148341)\end{array}$ & $\begin{array}{c}0.197432 \\
(0.151597)\end{array}$ \\
\hline$\Delta \mathbf{i}_{\mathrm{t}-2}$ & $\begin{array}{c}0.238371 \\
(0.147293)\end{array}$ & $\begin{array}{c}0.257638 \\
(0.129302)\end{array}$ & $\begin{array}{c}0.309427 * * \\
(0.130934)\end{array}$ & $\begin{array}{l}- \\
-\end{array}$ & - & - \\
\hline$y_{t}$ & $\begin{array}{c}0.004607 \\
(0.008286)\end{array}$ & - & $\begin{array}{c}0.022853 * * * \\
(0.006325)\end{array}$ & - & - & - \\
\hline$y_{t-1}$ & $\begin{array}{c}0.023728 \\
(0.012410)\end{array}$ & $\begin{array}{c}0.027152 * * * \\
(0.008539)\end{array}$ & & $\begin{array}{c}0.029212 * * \\
(0.011178)\end{array}$ & $\begin{array}{c}0.032103 * * * \\
(0.011806)\end{array}$ & $\begin{array}{c}0.027804 * * \\
(0.011218)\end{array}$ \\
\hline$\pi_{t}$ & $\begin{array}{c}0.464204 \\
(0.748669)\end{array}$ & $\begin{array}{l}- \\
-\end{array}$ & $\begin{array}{l}- \\
-\end{array}$ & $\begin{array}{l}- \\
-\end{array}$ & $\begin{array}{l}- \\
-\end{array}$ & $\begin{array}{l}- \\
-\end{array}$ \\
\hline$\pi_{\mathrm{t}-1}$ & $\begin{array}{c}0.361144 \\
(0.897027) \\
\end{array}$ & $\begin{array}{l}- \\
-\end{array}$ & $\begin{array}{l}- \\
-\end{array}$ & - & $\begin{array}{c}1.014320 \\
(0.915145)\end{array}$ & $\begin{array}{c}0.528870 \\
(0.829230) \\
\end{array}$ \\
\hline$\pi_{t}^{\mathrm{e}}$ & $\begin{array}{l}-1.125552 \\
(0.813869)\end{array}$ & - & $\begin{array}{l}- \\
-\end{array}$ & - & - & - \\
\hline $\bar{\pi}_{\mathrm{t}-1}^{\mathrm{e}}$ & $\begin{array}{l}1.910489 * * \\
(0.890481)\end{array}$ & $\begin{array}{c}1.891580^{* *} \\
0.907078\end{array}$ & $\begin{array}{c}2.055530 * * \\
(0.967035)\end{array}$ & $\begin{array}{l}1.926226^{* *} \\
(0.823525)\end{array}$ & - & $\begin{array}{c}1.746855 * * \\
(0.814707)\end{array}$ \\
\hline $\mathrm{R}^{2}$ ajust. & 0.376110 & 0.402240 & 0.387426 & 0.338351 & 0.307079 & 0.331143 \\
\hline$\overline{A I C}$ & 0.786829 & 0.688519 & 0.712999 & 0.794585 & 0.840767 & 0.819864 \\
\hline SIC & 1.092991 & 0.858609 & 0.883089 & 0.929515 & 0.975697 & 0.988526 \\
\hline HQ & 0.907244 & 0.755417 & 0.779896 & 0.847741 & 0.893923 & 0.886308 \\
\hline LM & 0.014 (1 lag) & 0.003 (1 lag) & $0.021(6 \operatorname{lgs})$ & $0.021(6 \operatorname{lgs})$ & $0.003(3 \operatorname{lgs})$ & $0.059(5 \operatorname{lgs})$ \\
\hline
\end{tabular}

Fonte: elaboração própria a partir das estatísticas do software econométrico utilizado.

Observa-se que nos modelos com a primeira diferença da meta de taxa Selic (modelos i a vi), os coeficientes de inércia da política monetária perdem significância estatística, em comparação com as especificações para o nível da meta Selic. Isso aponta para o fato de que a suavização da taxa Selic, no Brasil 
recente, refere-se às decisões quanto ao nível da taxa, formado a cada reunião do COPOM, porém não ao viés dessa taxa ou à sua tendência de variação imposta a cada reunião. Por sua vez, o gap do produto, embora com significância na maioria dos modelos, não possui poder explicativo considerável sobre a variação da taxa Selic, pari passu com o que se observa nas regras em nível.

$\mathrm{O}$ desvio da inflação face à meta inflacionária também não possui significância estatística nos modelos i a vi, do mesmo modo como observado nos modelos 1 a 6 . Isto reforça a constatação de que o BCB não forma o nível e a variação da taxa Selic com base nos desvios inflacionários já ocorridos, o que seria o caso sob um padrão backward-looking de política monetária. Tanto para o nível quanto para a variação da taxa Selic, as evidências são de que as expectativas de inflação 12 meses à frente, face à meta de inflação, é que têm poder explicativo no processo decisório do BCB. Os coeficientes do componente expectacional variam entre 1.74 e 2.05, todos com significância para a primeira defasagem.

Os dados reforçam a idéia de que o Banco Central brasileiro dá especial atenção às projeções de inflação futura como informação relevante no que diz respeito aos balanços de riscos inflacionários no médio e longo prazo. Comportamento este em linha com a proposição de que as autoridades monetárias devem mirar uma espécie de meta de expectativa inflacionária (Svensson, 1997), ancorando um comportamento forward-looking como estratégia ótima de política.

\section{RELAÇÕES ENTRE EXPECTATIVAS DE INFLAÇÃO E IN- FLAÇÃ̃O CORRENTE: UMA BREVE ANÁLISE EM VAR}

A idéia de que no período amostral estudado as mudanças das expectativas de inflação têm sido o principal fator explicativo das decisões de política monetária, no Brasil recente, pode conduzir a uma pergunta, qual seja: em que medida a dinâmica corrente e passada da inflação está determinando a formação de expectativas inflacionárias 12 meses à frente? Ou seja, há um padrão adaptativo de formação de expectativas de inflação na economia brasileira? No caso de uma resposta afirmativa - as expectativas de inflação são causadas pela inflação observada - pode-se sugerir que a melhor forma do BCB impor controle à inflação esperada é por meio do controle da própria inflação observada, ou seja, "disciplinando" as expectativas. 
Ademais, seria interessante examinar se a dinâmica das expectativas de inflação está afetando significativamente o comportamento da inflação corrente, o que, em caso afirmativo, apontaria para uma curva de Phillips forward-looking na economia brasileira recente, contrariando a especificação da equação 2 neste trabalho. Em suma, trata-se de analisar as relações de interdependência entre ambas as variáveis no período estudado.

Isto pode ser feito através da adoção de um modelo de Vetores Auto-Regressivos (VAR) bivariado, em que cada variável é dependente face a valores defasados de si mesma e da outra variável ${ }^{9}$. Os modelos VAR são de grande interesse em trabalhos de macroeconomia aplicada, uma vez que permitem o estudo dos efeitos distribuídos temporalmente sofridos por determinada variável, quando de um choque nas outras variáveis do modelo. Ao contrário das análises de regressão por mínimos quadrados ordinários (MQO), que identificam a resposta esperada ou média, no período amostral, de uma variável às mudanças nas demais; os modelos VAR identificam a dinâmica temporal daquela resposta dentro de um intervalo de tempo especificado. Isto é de extrema utilidade porque as respostas de uma variável, às mudanças nas demais, podem variar em magnitude e natureza ao longo do tempo.

As etapas são as seguintes: i) definição da melhor defasagem do VAR; b) análise de ordenamento de Cholesky, através dos testes de causalidade Granger ${ }^{10}$; c) análise da decomposição da variância e das funções de impulso-resposta de cada variável, etapa de extração do significado econômico.

Com base nos critérios de Akaike (AIC), Schwarz (SIC) e Hannan-Quinn (HQ), pode-se escolher o modelo VAR(1) com constante, como sendo o de melhor ajuste aos dados (menores valores para todos os critérios). A Tabela 5 apresenta as estatísticas para cada defasagem testada, com e sem constante.

\footnotetext{
Neste trabalho, não aplicaremos um VAR a todas as variáveis das regras de política especificadas na seção 4; tal análise, embora de efeitos potenciais positivos, estenderia em demasia o assunto, ficando para outro espaço de discussão.

10 Segundo Cavalcanti (20 l 0), a identificação do ordenamento do modelo VAR através da causalidade Granger não seria apropriada, a princípio. Isto porque a ordenação de Cholesky indica uma causalidade contemporânea entre as variáveis, ao passo que os testes de Granger indicam uma causalidade de precedência temporal. No entanto, o autor abre uma possibilidade para o uso dos testes Granger como método de ordenamento de Cholesky: a premissa de que há uma correlação positiva entre a probabilidade de causalidade Granger e causalidade contemporânea entre as variáveis, ainda que esta premissa possa não ser verificada em determinados casos macroeconômicos. No entanto, o presente trabalho adota os testes Granger como método de ordenamento assim como o fazem uma série de trabalhos em macro aplicada ao Brasil, a exemplo de Oreiro et. al. (2006) Mendonça (2005) e Mendonça (2004)
} 
Em seguida, como de costume em uma série de trabalhos recentes em macroeconomia aplicada ao Brasil - conferir, por exemplo, Mendonça (2004), Oreiro et. al. (2006) e Mendonça (2005) - realizam-se testes de causalidade Granger a fim de se identificar o ordenamento de Cholesky no modelo VAR, ou seja, o encadeamento de causa e efeito do modelo, etapa importante antes de se proceder à análise da decomposição da variância e das funções impulso-resposta (Mendonça, 2005).

A Tabela 6 apresenta os resultados (estatística Qui-quadrado e p-valor) e mostra que a hipótese nula de não causa-Granger pode ser rejeitada para a variável $\pi$, ou seja, a inflação corrente afeta a expectativas de inflação com precedência temporal; por sua vez, aceita-se a hipótese nula de não causa-Granger para a variável $\pi^{\mathrm{e}}$ : as expectativas de inflação, no período amostral, não afetam a inflação corrente com precedência temporal.

Tabela 5 - Critério de adoção do n. de defasagens para o VAR

Com constante

\begin{tabular}{cccc}
\hline Lag & AIC & SC & HQ \\
\hline $\mathbf{0}$ & -6.110411 & -6.0406 & -6.083104 \\
\hline $\mathbf{1}$ & $-6.440482^{*}$ & $-6.231048^{*}$ & $-6.358561^{*}$ \\
\hline $\mathbf{2}$ & -6.376423 & -6.027365 & -6.239887 \\
\hline $\mathbf{3}$ & -6.263623 & -5.774943 & -6.072473 \\
\hline $\mathbf{4}$ & -6.193351 & -5.565048 & -5.947587 \\
\hline $\mathbf{5}$ & -6.086658 & -5.318732 & -5.78628 \\
\hline $\mathbf{6}$ & -5.977145 & -5.069596 & -5.622153 \\
\hline
\end{tabular}

(*) indicando a melhor estatística
Sem constante

\begin{tabular}{cccc}
\hline Lag & AIC & SC & HQ \\
\hline $\mathbf{1}$ & -6.098565 & $-5.958942 *$ & $-6.043951^{*}$ \\
\hline $\mathbf{2}$ & -6.055402 & -5.776156 & -5.946174 \\
\hline $\mathbf{3}$ & -6.069443 & -5.650574 & -5.9056 \\
\hline $\mathbf{4}$ & $-6.101375^{*}$ & -5.542883 & -5.882918 \\
\hline $\mathbf{5}$ & -6.045355 & -5.347241 & -5.772284 \\
\hline $\mathbf{6}$ & -5.966421 & -5.128683 & -5.638735 \\
\hline
\end{tabular}

(*) indicando a melhor estatística Fonte: elaboração própria a partir das estatísticas do software econométrico utilizado.

Tabela 6 - Teste de causalidade Granger para o VAR(1)

\begin{tabular}{ccc|ccc}
\hline \multicolumn{6}{c}{ Variável dependente } \\
\hline$\pi^{\mathrm{e}}$ & & \multicolumn{4}{c}{$\pi$} \\
\hline Causa & Chi-sq & p-valor & Causa & Chi-sq & p-valor \\
\hline$\pi$ & 12.48876 & 0.0004 & $\pi^{\mathrm{e}}$ & 0.857209 & 0.355 \\
\hline
\end{tabular}

Fonte: elaboração própria a partir das estatísticas do software econométrico utilizado.

Com base nessas informações, adota-se a ordenação de Cholesky $\pi-\pi^{\mathrm{e}}$ (leia-se: inflação causa expectativa de inflação). A partir disto, realizam-se 
as análises de decomposição da variância de cada variável e das funções impulso-resposta, na Tabela 7 e 8, e na Figura 1, respectivamente.

Em relação à inflação corrente, verifica-se que a quase totalidade de sua variância (98.68\%) é explicada pela própria variável, mesmo após 12 meses (Tabela 7) de ocorrência de um choque, ou seja, as expectativas de inflação têm uma influência insignificante sobre as flutuações da inflação corrente ao longo do tempo estudado. Pelas funções de impulso-resposta (Figura 1), observa-se que choques na inflação corrente geram fortes efeitos na dinâmica da própria variável, pelo componente de inércia, e aqueles efeitos são diluídos apenas lentamente ao longo do tempo, após o $6^{\circ}$ a $7^{\circ}$ mês aproximadamente. Já os choques de expectativas de inflação não possuem influência estatisticamente significante sobre a dinâmica da inflação corrente (indicado pelo teste Granger). Após 12 meses, apenas $1.3 \%$ de sua variância é devido a flutuações nas expectativas de inflação (Tabela 7).

\begin{tabular}{|c|c|c|c|}
\hline \multicolumn{4}{|c|}{ Decomposição da Variância - $\pi$} \\
\hline Período & S.E. & $\pi^{\mathrm{e}}$ & $\pi$ \\
\hline 1 & 0.045295 & 0 & 100 \\
\hline 2 & 0.049397 & 0.944275 & 99.05573 \\
\hline 3 & 0.050696 & 1.233417 & 98.76658 \\
\hline 4 & 0.050999 & 1.299912 & 98.70009 \\
\hline 5 & 0.051062 & 1.313642 & 98.68636 \\
\hline 6 & 0.051074 & 1.316349 & 98.68365 \\
\hline 7 & 0.051077 & 1.316872 & 98.68313 \\
\hline 8 & 0.051077 & 1.316973 & 98.68303 \\
\hline 9 & 0.051077 & 1.316992 & 98.68301 \\
\hline 10 & 0.051077 & 1.316996 & 98.683 \\
\hline 11 & 0.051077 & 1.316996 & 98.683 \\
\hline 12 & 0.051077 & 1.316996 & 98.683 \\
\hline
\end{tabular}

Em relação a esta última variável, sua decomposição de variância (Tabela 8) indica que a inflação corrente possui forte poder de explicação. Após 12 meses de um choque observado, ainda 24\% aproximadamente 
são devidos a flutuações na inflação corrente, demonstrando um comportamento relativamente adaptativo das expectativas de inflação no país. Pelas funções impulso (Figura 1), pode-se notar que, após um choque na inflação corrente, as expectativas sofrem resposta relevante e retornam para seus níveis normais apenas após 7 a 8 meses aproximadamente. Por outro lado, a dinâmica das expectativas após um choque em si mesmas apresenta retorno mais acelerado para seus níveis normais, logo após o $2^{\circ}$ a $3^{\circ}$ mês aproximadamente.

Tabela 8 - Decomposição da variância da inflação esperada

\begin{tabular}{cccc}
\hline \multicolumn{4}{c}{ Decomposição da Variância $-\boldsymbol{\pi}^{\mathrm{e}}$} \\
\hline Período & S.E. & $\boldsymbol{\pi}^{\mathrm{e}}$ & $\boldsymbol{\pi}$ \\
\hline $\mathbf{1}$ & 0.050263 & 95.27377 & 4.72623 \\
\hline $\mathbf{2}$ & 0.057299 & 80.31496 & 19.68504 \\
\hline $\mathbf{3}$ & 0.058776 & 76.40652 & 23.59348 \\
\hline $\mathbf{4}$ & 0.059073 & 75.57642 & 24.42358 \\
\hline $\mathbf{5}$ & 0.05913 & 75.41012 & 24.58988 \\
\hline $\mathbf{6}$ & 0.059142 & 75.3777 & 24.6223 \\
\hline $\mathbf{7}$ & 0.059144 & 75.37145 & 24.62855 \\
\hline $\mathbf{8}$ & 0.059144 & 75.37026 & 24.62974 \\
\hline $\mathbf{9}$ & 0.059144 & 75.37003 & 24.62997 \\
\hline $\mathbf{1 0}$ & 0.059144 & 75.36999 & 24.63001 \\
\hline $\mathbf{1 1}$ & 0.059144 & 75.36998 & 24.63002 \\
\hline $\mathbf{1 2}$ & 0.059144 & 75.36998 & 24.63002 \\
\hline
\end{tabular}

Fonte: elaboração própria a partir das estatísticas do software econométrico utilizado. 


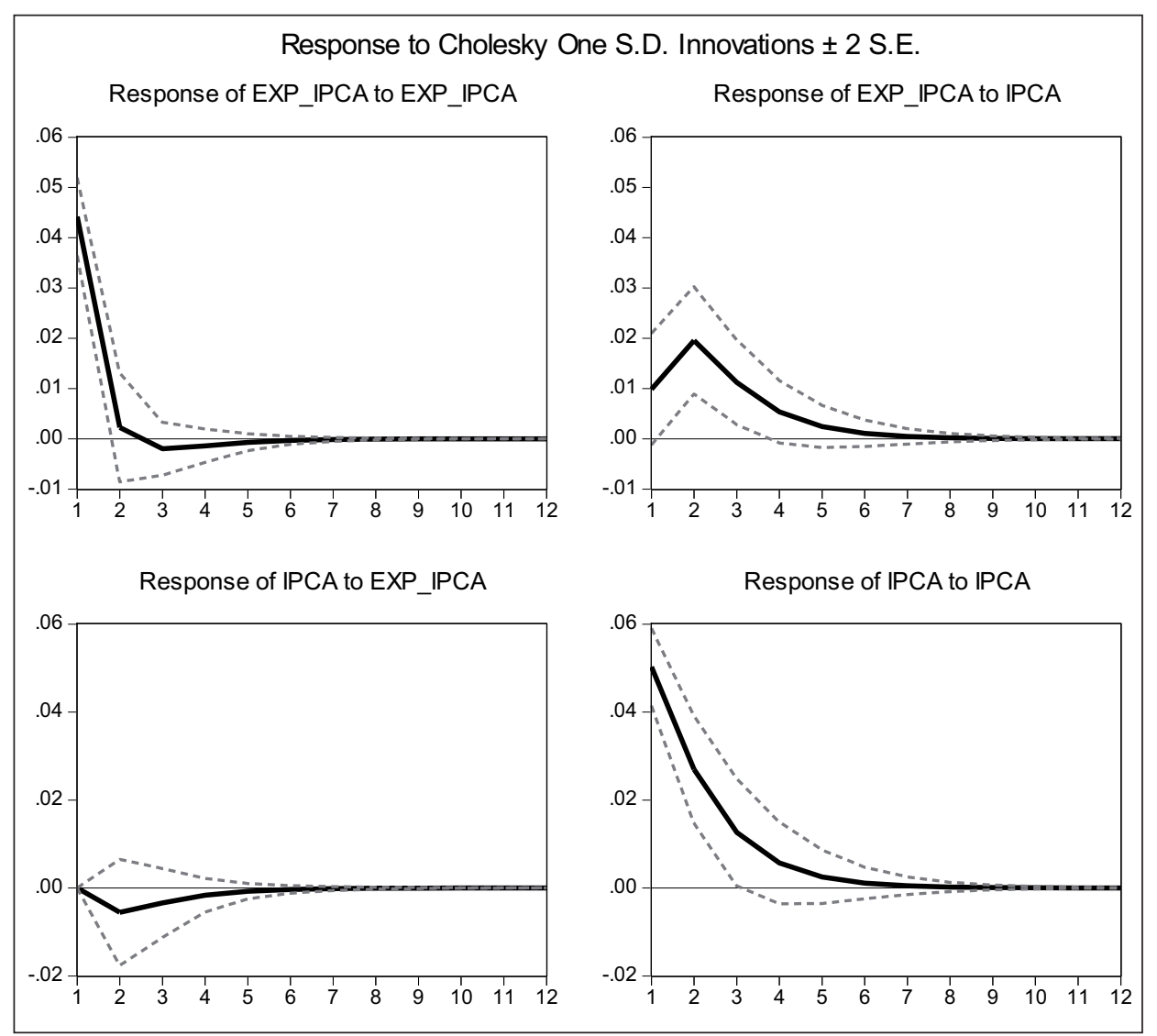

Figura 1 - Funções impulso-resposta. EXP_IPCA $=\pi^{\mathrm{e}}$; IPCA $=\pi$. Fonte: saídas do software econométrico utilizado.

\section{CONSIDERAÇÕES FINAIS E PESQUISA FUTURA}

O presente trabalho teve como finalidade estudar a aderência empírica de algumas especificações de regras de política monetária na economia brasileira recente. A proposição de que os bancos centrais devem dar atenção especial à dinâmica das expectativas inflacionárias - ao decidirem os ajustes nas metas de taxas de juros de curto prazo - parece estar sendo seguida fortemente pela autoridade monetária brasileira, tanto em relação aos níveis quanto às variações da taxa Selic. 
Por outro lado, não foram encontradas evidências de que o gap do produto possua impactos expressivos na formação das taxas de juros; entretanto, esse resultado pode estar relacionado à composição da variável gap do produto utilizada neste trabalho. Proxies alternativas podem ser aplicadas em futuros trabalhos a fim de que aquelas evidências sejam melhor avaliadas. Por sua vez, verificou-se forte inércia no nível da meta de taxa de juros, decidida pelo $\mathrm{BCB}$, o que corrobora a preferência por suavização dos juros, fato estilizado pela literatura atual.

Ademais, observou-se um comportamento adaptativo na formação das expectativas de inflação, principal variável na explicação das decisões de política monetária; ao passo que a dinâmica da inflação observada mostrouse altamente inercial: no período amostral, as expectativas de inflação não possuem impacto estatisticamente significante sobre a dinâmica corrente dos preços, o que aponta para uma espécie de curva de Phillips backward -looking na economia brasileira recente.

Sugere-se que o BCB ainda precisa domar sistematicamente a inflação corrente como forma de controlar as expectativas, ou seja, a desejada ancoragem dessa última, a qual advém de um contexto de consolidação de credibilidade, parece ainda não ter sido alcançada pelo BCB.

Finalmente, a presente pesquisa pode desdobrar-se em alguns caminhos interessantes, como pela especificação de outras regras a serem testadas e pela adoção de métodos econométricos alternativos. Em especial, propõe-se a aplicação de um Vetor Auto-Regressivo a partir de todas as variáveis usadas neste artigo, a fim de que as dinâmicas de cada variável, face às flutuações nas demais, sejam analisadas, o que pode complementar as evidências por ora encontradas.

\section{BRAZILIAN CENTRAL BANK'S RULE ON INERTIAL INFLA- TION AND ADAPTIVE EXPECTATION: FROM JAN-2005- JUN2010}

Abstract

From the theoretical framework of Taylor rules, this work studies the empirical adhesion of alternative monetary policy rules in the Brazilian 
economy, from January 2005 to June 2010. The main results show: a) the presence of strong inertia level of the Selic rate, b) inflation expectations as a highly explanatory variable in composing levels and variations of Selic rate, suggesting forward-looking behavior of the Central Bank, c) product gap with low explanatory power in monetary policy decisions, d) inflation expectations with strong adaptive component in the studied period.

Keywords: Inflationary Expectations, Taylor Rules, Brazilian Central Bank.

JEL Classification: E17; E31; E52.

\section{REFERÊNCIAS BIBLIOGRÁFICAS}

Ball, L. (1999). "Efficient rules for monetary policy". International Finance, v. 2, n. 1:63-83.

Barbosa, F. H. \& Soares, J.J.S. (2006). Regra de Taylor no Brasil: 19992005. In: Encontro da Anpec.

Blinder, A. S. (2006). "Monetary policy today: sixteen questions and about twelve answers". CEPS working papers, $\mathrm{n}^{\mathrm{o}} 129$.

Bogdanski, J; A. A. Tombini \& R. C. Werlang (2000). "Implementing inflation targeting in Brazil", Working Paper Series, no 1, Central Bank of Brazil, July.

Box, G. E. P. \& Jenkins, F. M. (1976). Time Series Analysis: Forecasting and Control, 2nd. ed. Oakland, CA: Holden-Day.

Cavalcanti, M. A. F. H. (2010). "Identificação de modelos VAR e causalidade de Granger: uma nota de advertência". Economia Aplicada, v.14(2): 251-260. 
Clarida, R., Galí, J, \& Gertler, M. (1999). "The science of monetary policy: a new Keynesian perspective". Journal of Economic Literature, v. XXXVII: 1661-1707.

Dikey, D. A. \& Fuller, W. A. (1979). "Distribution of the estimador for autoregressive time series with a unit root". Journal of the American Statistical Association, Boston, v. 74, n. 366: 427-431.

Dikey, D. A. \& Fuller, W. A. (1981). "Likelihood ratio statistics for autoregressive time series with a unit root". Econometrica, Menasha, v. 49, n. 4, pp. 1057-1072.

Enders, W. (2004). Applied econometric time series. New York: John Wiley\& Sons, 2004, 2a ed., pp. 466.

Fuller, W. A. (1976). Introduction to statistical time series. New York: John Wiley \& Sons.

Galí, J. \& Gertler, M. (2007). "Macroeconomic Modeling for Monetary Policy Evaluation”. Journal of Economic Perspectives, 21: 25-45.

Granger, C. \& Newbold, P. (1974). Spurious Regression in Econometrics. Journal of Econometrics, 2: 111-120.

Haldane, A. G. \& Batini, N. (1998). Forward-looking rules for monetary policy". NBER Working Papers Series, n. 6543.

Hill, R. C., Griffiths W. E., Judge, G. G. (1998). Undergraduate Econometrics, New York, John Wiley \& Sons.

Hodrick, R. J. \& Prescott, E. C. (1997). "Postwar U.S. business cycles: an empirical investigation". Journal of Money Credit and Banking,n. 29: 1-16.

Holland, M. (2005). Monetary and exchange rate policy in Brazil after inflation targeting. Berkeley: University of California. 
Kwiatkowski, D.; Phillips, P. C. B.; Schmidt, P. \& Shin, Y. (1992). "Testing the null hypothesis of stationarity against the alternative of a unit root: How sure are we that economic time series have a unit root?". Journal of Econometrics, 54(1-3): 159-178.

Maddala, G. S. (1992). Introduction to econometrics, 2nd edition. New York: MacMillan.

Mendonça, H. F. (2004). “Dívida pública e estabilidade de preços no período pós-real: Explorando relações empíricas”. Estudos Econômicos 34, 345-368.

Mendonça, H. F. (2005). "Metas para inflação e variáveis macroeconômicas: uma avaliação empírica”. In: Encontro Nacional da ANPEC.

Minella, A.; de Freitas, P.S.; Goldfajn, I. \& Muinhos, M.K. (2003). "Inflation targeting in Brazil: lessons and challenges”. BIS Papers, n. 19: 106-133.

Oreiro, J. L., Paula, L. F., Ono, F. H. \& Silva, G. J. C. (2006). "Determinantes macroeconômicos do spread bancário no brasil: teoria e evidência recente.". Economia Aplicada 10, 609-634.

Svensson, L. E. O. (1997). "Inflation forecast targeting: implementing and monitoring inflation targets”. European Economic Review, 41: 1111-1146.

Svensson, L. E. O. (1999). "Inflation targeting as a monetary policy rule". Journal of Monetary Economics, 43 (June): 607-54.

Taylor, J. B. (1993). “Discretion versus Policy Rules in Practice”. CarnegieRochester Conference Series Public Policy, 39: 195-214.

Woodford, M. (2003). "Interest and Prices: Foundations of a Theory of Monetary Policy”. Princeton University Press.

Wooldridge, J. M. (2009). Introductory Econometrics: A Modern Approach, South-Western College Publishing. 


\section{ANEXO - COMPORTAMENTO GRÁFICO DAS SÉRIES}

Gráfico 1 - i $\mathrm{t}_{\mathrm{t}}(\mathrm{jan} / 2005$ - jun/2010)

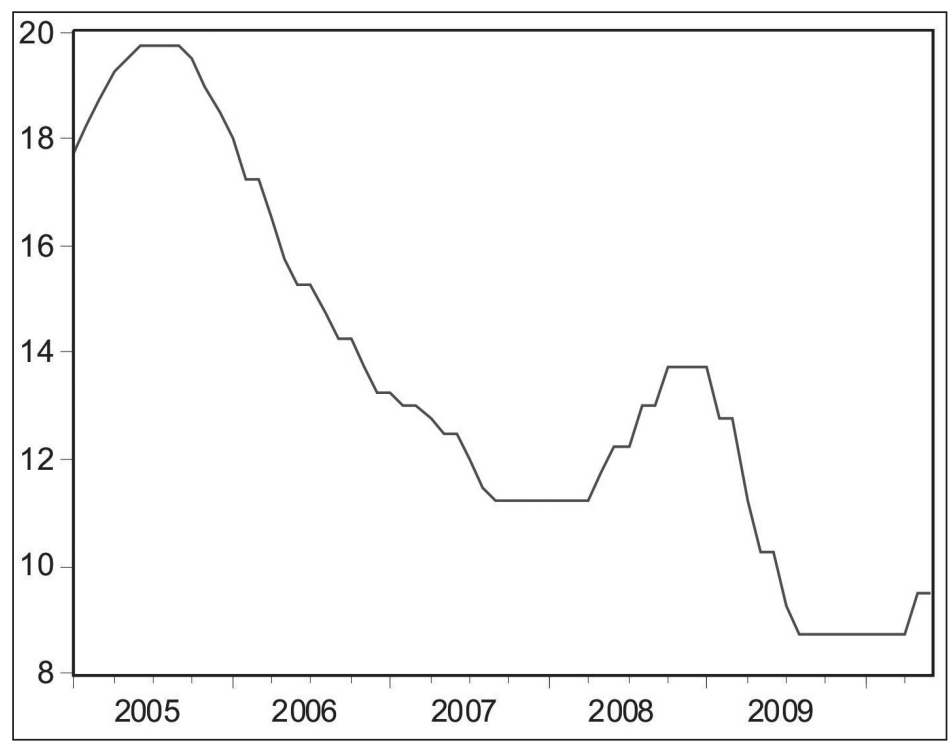

Fonte: saída do pacote E-views.

Gráfico $2-\pi_{\mathrm{t}}(\mathrm{jan} / 2005-\mathrm{jun} / 2010)$

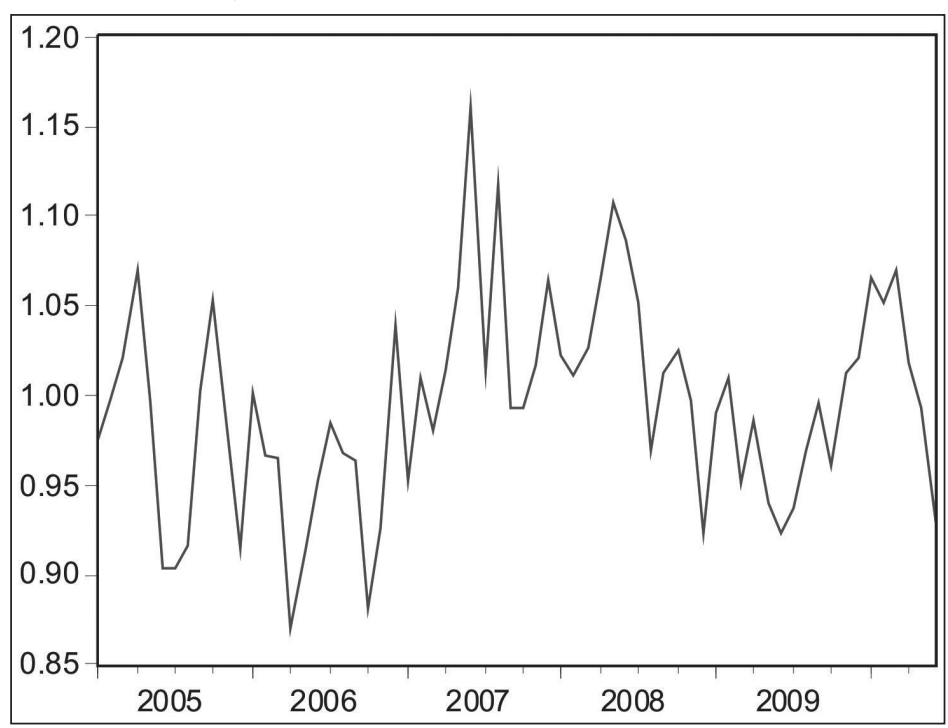

Fonte: saída do pacote E-views. 
Gráfico $3-\pi_{\mathrm{t}}^{\mathrm{e}}(\mathrm{jan} / 2005-\mathrm{jun} / 2010)$

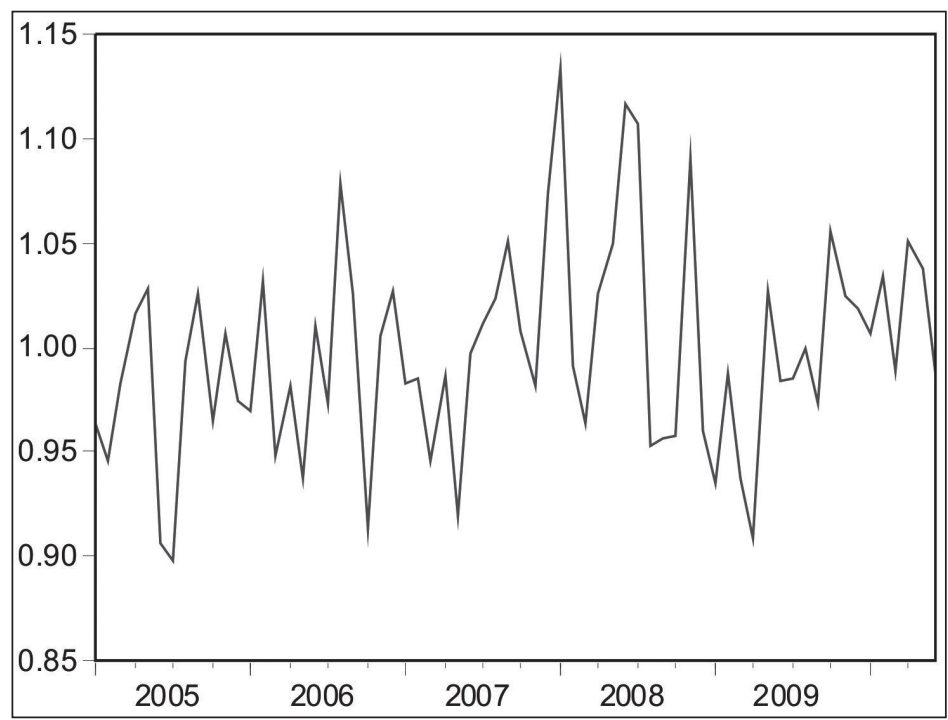

Fonte: saída do pacote E-views.

Gráfico $4-\mathrm{y}_{\mathrm{t}}(\mathrm{jan} / 2005-\mathrm{jun} / 2010)$

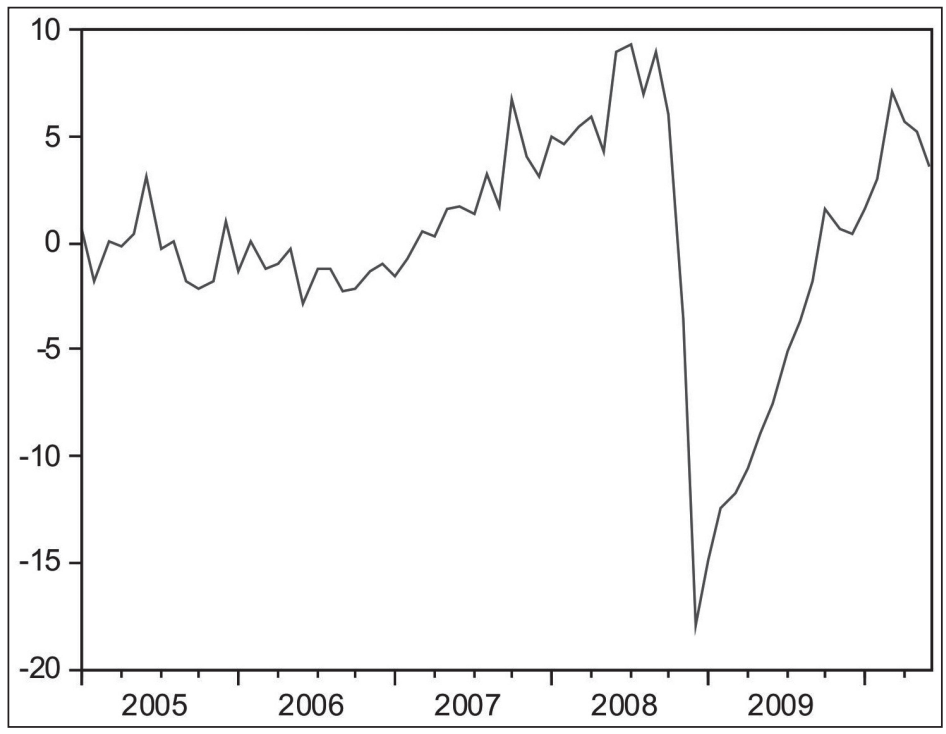

Fonte: saída do pacote E-views. 